\title{
Atypical time-dependent magnetic behaviors of Fe-Bi films
}

\author{
Jen-Hwa Hsua) \\ Department of Physics, National Taiwan University, Taipei 106, Taiwan and Center for Nanostorage \\ Research, National Taiwan University, Taipei 106, Taiwan
}

Hong-Xian Wang, An-Cheng Sun, and Po-Cheng Kuo

Institute of Materials Science and Engineering, National Taiwan University, Taipei 106, Taiwan

(Presented on 3 November 2005; published online 20 April 2006)

\begin{abstract}
Films of metastable Fe-Bi solid solution were prepared by dc sputtering on $\mathrm{Si}(100)$ substrate. The magnetic properties of Fe-Bi films are generally changing slowly with aging time. The as-prepared films exhibit a large out-of-plane magnetic anisotropy. The natural aging process precipitates out Fe atoms and forms nanograins with dead magnetic layers on the surface, reducing saturation magnetization and eliminating the magnetic anisotropy of the as-prepared films. On the contrary, Co-Bi films do not exhibit this weakly time-dependent behavior. (C) 2006 American Institute of Physics. [DOI: 10.1063/1.2173605]
\end{abstract}

\section{INTRODUCTION}

Our previous studies demonstrated a very long magnetic relaxation time at room temperature in the $\mathrm{Fe}-\mathrm{Bi}$ systems. ${ }^{1}$ This unique characteristic is attributable to the large spinorbital splitting of $\mathrm{Bi}$, which prevents the magnetic element from rapidly releasing its magnetic energy to the system. Honda and Nagata recently discovered an enhanced Hall coefficient of $5.25 \times 10^{-10} \Omega \mathrm{cm} / \mathrm{G}$ in the $\mathrm{Co}-\mathrm{Bi}$ film, which exceeds that of pure Co bulk by two orders of magnitude. ${ }^{2}$ Hence, the combination of magnetic metal and semimetallic bismuth gives rise to numerous peculiar and interesting results, because Bi has several unique physical properties. For instance, the Fermi wavelength of the semimetallic $\mathrm{Bi}$ is as large as $400 \AA$, in contrast to a few angstroms for most metals. ${ }^{3}$ The carrier's mean free path in $\mathrm{Bi}$ can be as long as a few millimeters at $4.2 \mathrm{~K}$, several orders of magnitude larger than in most metals. This research continues work on the Bi-based sample system and examines the timedependent magnetic behavior of $\mathrm{Fe}-\mathrm{Bi}$. The $\mathrm{Co}-\mathrm{Bi}$ system is also examined for comparison.

\section{EXPERIMENT}

$\mathrm{Fe}_{x} \mathrm{Bi}_{1-x}$ and $\mathrm{Co}_{x} \mathrm{Bi}_{1-x}$ ( $x$ represents the relative volume fraction of the $\mathrm{Fe}$ and Co contents) films were fabricated by dc magnetron sputtering using composite targets made from the mixture of $\mathrm{Bi}$ and $\mathrm{Fe}$ or Co powders. The $\mathrm{Si}(100)$ wafers were used as substrates. The thickness of the films was approximately $1 \mu \mathrm{m}$. The composition of the films was verified using an electron probe x-ray microanalyzer (EPMA). The difference between the nominal and actual compositions was under $10 \%$. The structure of the film was determined by $\mathrm{x}$-ray powder diffraction (XRD) and the film morphology was elucidated by transmission electron microscopy (TEM). Magnetic measurements were made using a vibrating sample

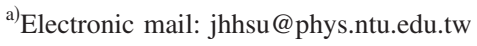

magnetometer (VSM) with a maximum applied field of 1.2 T. The samples were kept in desiccators between measurements to prevent oxidation.

\section{RESULTS AND DISCUSSION}

Figure 1 presents the XRD patterns of $\mathrm{Fe}-\mathrm{Bi}$ and $\mathrm{Co}-\mathrm{Bi}$ films. In Fig. 1(a), the XRD patterns of the as-prepared samples clearly indicate the presence of only the rhombohedral phase of bismuth. No diffraction peaks that correspond to either $\mathrm{Fe}$ or $\mathrm{Co}$ are found in the pattern for any concentration studied. Figure 1(a) also compares the Bragg angles of $\mathrm{Fe}-\mathrm{Bi}$ with those of a pure bismuth film. A slight $\mathrm{Bi}(006)$ peak shift is observed. If $\mathrm{Fe}$ and $\mathrm{Bi}$ atoms are present substitutionally or interstitially and form a solid solution during the sputtering process, then the large difference between the atomic radii of $\mathrm{Fe}$ and $\mathrm{Bi}\left(r_{\mathrm{Fe}}=1.26 \AA\right.$ and $\left.r_{\mathrm{Bi}}=1.70 \AA\right)$ causes distortion of the bismuth lattice. Furthermore, the change in the $d$ spacing between the crystal planes shifts the diffraction angle in the XRD pattern. A comparison of the Bragg angles of $\mathrm{Fe}-\mathrm{Bi}$ and pure bismuth films, according to the Bragg law $(n \lambda=2 d \sin \theta)$, reveal that Fe atoms preferentially replace $\mathrm{Bi}$ atoms on $\mathrm{Bi}(006)$ crystal planes. This result also yields a compression ratio of $0.996\left[d_{\mathrm{FeBi}(h k l)} / d_{\mathrm{Bi}(h k l)}\right]$ for the distance between planes. Figure 1(b) presents the representative XRD patterns of the as-prepared and aged samples. Aging does not markedly change the XRD patterns. A more detailed discussion is presented below.

Figure 2(a) displays the electron diffraction pattern of the as-prepared $\mathrm{Fe}_{0.4} \mathrm{Bi}_{0.6}$ sample. The pattern has sharp rings and confirms that the film has a rhombohedral structure. Figures 2(b) and 2(c) are the bright field images of the asprepared $\mathrm{Fe}_{0.4} \mathrm{Bi}_{0.6}$ film and that aged for 20 days, respectively. They are shown using a negative film to improve the contrast. Bismuth grains of various sizes are present in the as-prepared film, while the sample aged for 20 days contains many nanograins that are distributed along the grain boundaries of $\mathrm{Bi}$, generally with a diameter under $3 \mathrm{~nm}$. From the TEM energy dispersive $\mathrm{x}$-ray spectrometer (EDS) analysis, we confirm that the nanograins are $\mathrm{Fe}$. Fe and $\mathrm{Bi}$ are com- 


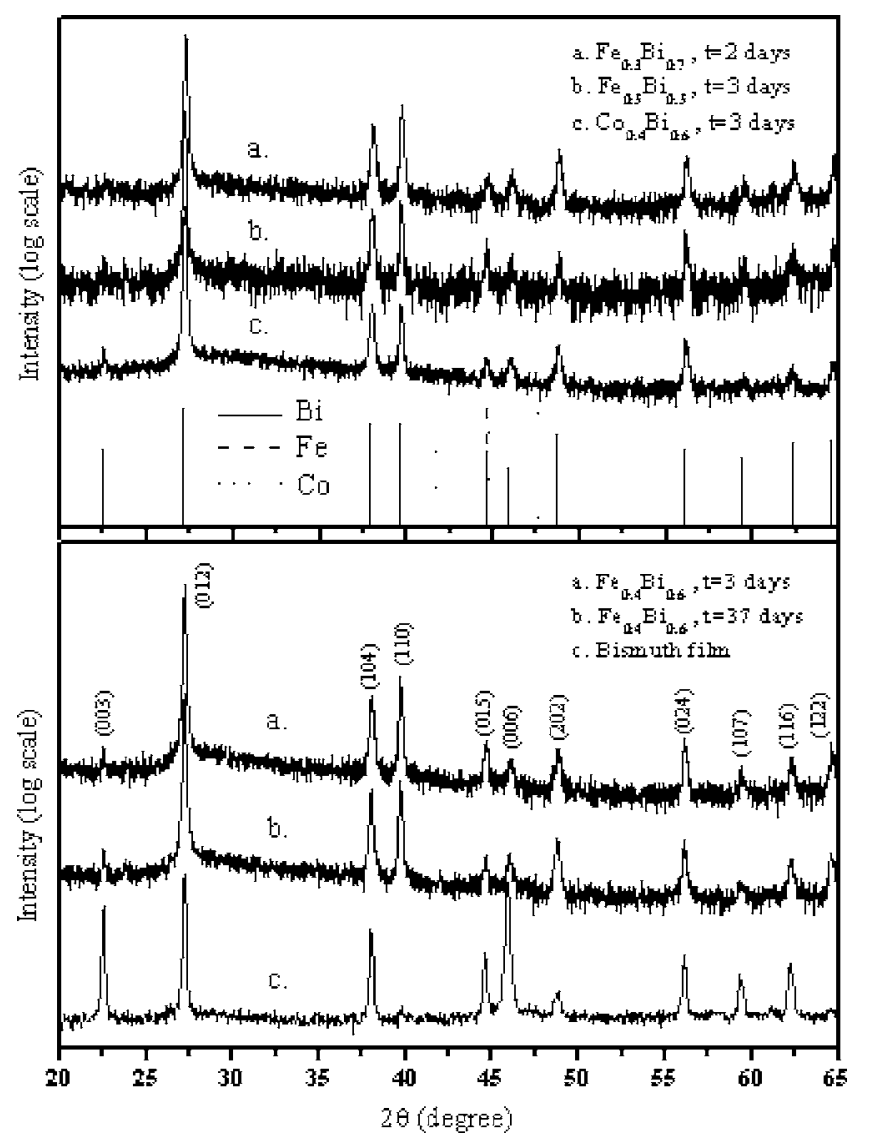

FIG. 1. (a) XRD patterns of $\mathrm{Fe}-\mathrm{Bi}$ and $\mathrm{Co}-\mathrm{Bi}$ films during the early stages of aging. (b) XRD patterns of $\mathrm{Fe}_{0.4} \mathrm{Bi}_{0.6}$ film at two aging periods. The diffraction pattern of the $\mathrm{Bi}$ film is also displayed for comparison.

pletely immiscible and no other intermetallic compounds are present; the Bi lattice with sites occupied by Fe atoms is in an unstable high-energy state. Spontaneous phase decomposition occurs to lower the Gibbs free energy of the system. ${ }^{4}$ This system has sufficient driving force to initiate precipitation even at room temperature. ${ }^{5}$ The natural aging effect at room temperature contributes to the ongoing precipitation of $\mathrm{Fe}$ at the grain boundaries and triggers changes in its physical characteristics. However, even when Fe precipitated, no change in the XRD patterns was detected after aging, as described earlier because the atomic number of $\mathrm{Bi}$ is large, so the atomic scattering factor is much higher than those of $\mathrm{Fe}$ and Co. Consequently, those peaks from Bi obscure the diffraction peaks produced by $\mathrm{Fe}$ and $\mathrm{Co}$ in the XRD patterns.

Figure 3 compares the $M-H$ loop of the $\mathrm{Fe}_{0.4} \mathrm{Bi}_{0.6}$ film aged for various aging periods and the insert presents the variation of the saturation field $\left(H_{s}\right)$ with aging periods. Due to the uncertainty in the determination of film thickness (the film surface is very rough because of the low melting point of $\mathrm{Bi}$ ), the ordinate of Fig. 3 was represented by the total magnetic moment $m$. The as-prepared Fe-Bi films exhibit a strong out-of-plane magnetic anisotropy. ${ }^{6}$ When an in-plane external magnetic field $(H / /$ film $)$ is applied to the film, below $H_{s}$, the hysteresis loops show that the magnetization is linearly dependent on the applied field. No linear relationship applies when the field is applied perpendicularly to the film.

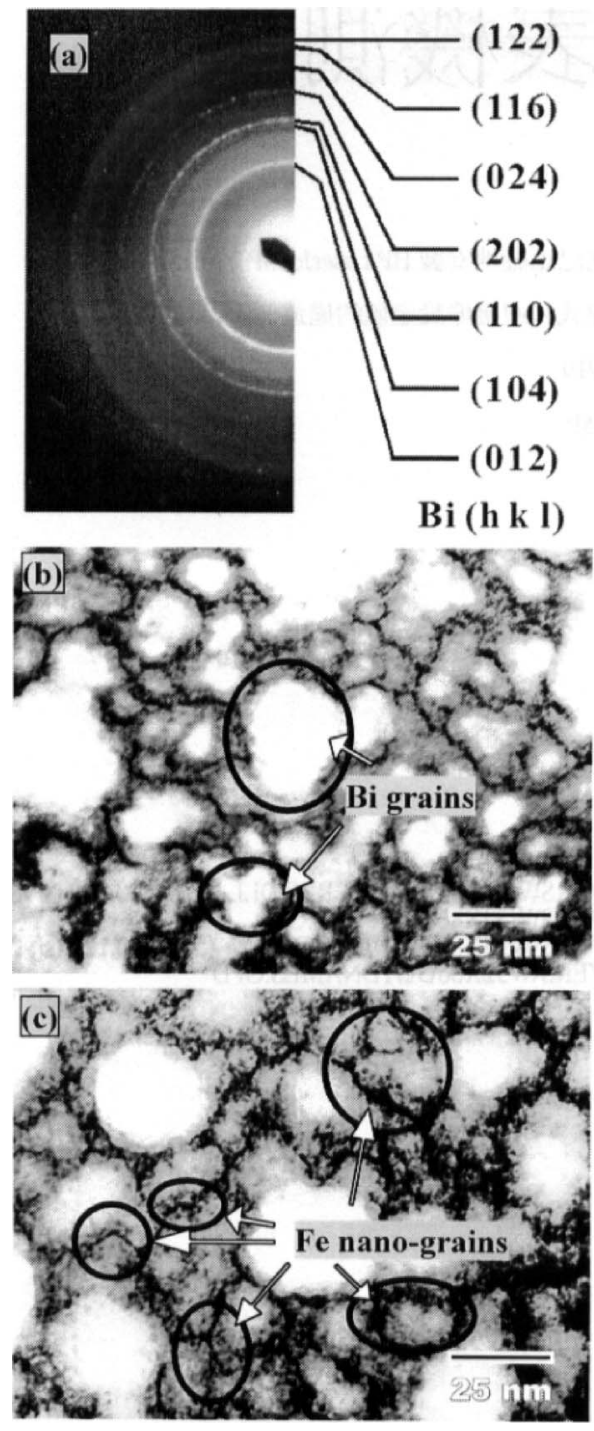

FIG. 2. (a) Electron diffraction ring pattern of as-prepared $\mathrm{Fe}_{0.4} \mathrm{Bi}_{0.6}$, (b) TEM bright-field micrographs (negative film) of the as-prepared $\mathrm{Fe}_{0.4} \mathrm{Bi}_{0.6}$, and (c) TEM bright-field micrographs (negative film) of $\mathrm{Fe}_{0.4} \mathrm{Bi}_{0.6}$ after aging for 20 days.

This anisotropy causes the saturation field $\left(H_{s}\right)$ of the asprepared sample to rise to $0.5 \mathrm{~T}$. Also the magnetic anisotropy energy rises with the increase in concentration of $\mathrm{Fe}$. At $x=0.5,0.4$, and 0.3 , the values of $H_{s}$ are $0.690,0.516$, and $0.336 \mathrm{~T}$, respectively, for the as-prepared samples. The strong magnetic anisotropy may originate from a preferred distribution of $\mathrm{Fe}$ atoms in some particular planes of the bismuth lattice. ${ }^{7,8}$ A textured polycrystalline film thus exhibits an outof-plane magnetic anisotropy in the Fe-Bi solid solution. As the duration increases, the anisotropy decreases and eventually vanishes, as shown in the insert of Fig. 3. The loop becomes a normal ferromagnetic hysteresis loop after a long period of natural aging precipitation. Furthermore, $H_{s}$ declines almost linearly with aging time, when $H /$ / film.

Figure 4 plots the variation of the saturation magnetization $\left(m_{s}\right)$, the remanence $\left(m_{r}\right)$, and the coercivity $\left(H_{c}\right)$ at different aging durations for $x=0.5,0.4$, and 0.3 . Regardless of Fe concentration, $m_{s}$ and $m_{r}$ decrease and $H_{c}$ rises with aging time. The changes are most drastic in the early stage 


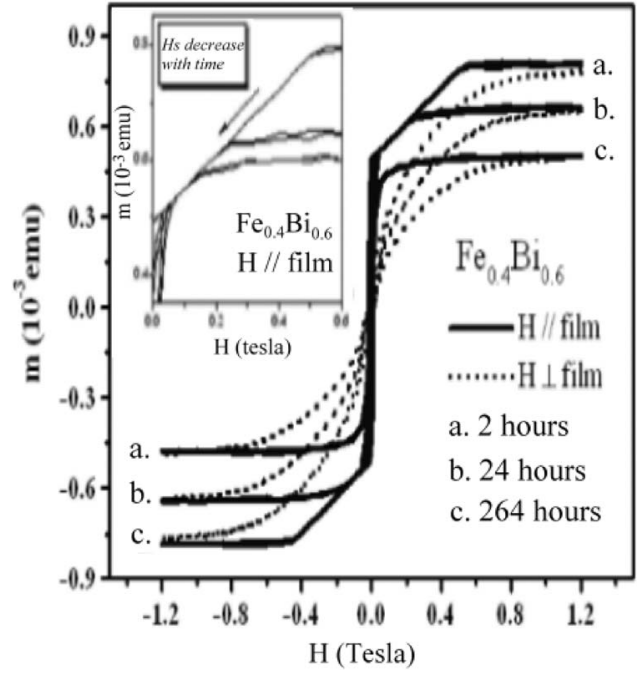

FIG. 3. Magnetization loops of $\mathrm{Fe}_{0.4} \mathrm{Bi}_{0.6}$ at room temperature at both field directions. The insert reveals that the saturation field $\left(H_{s}\right)$ shifts to a lower field during aging with the in-plane field $(H / /$ film)

and tend to stabilize after about a month. The aging process is characterized by time-dependent magnetic properties. The precipitated Fe grains are very small and the surface properties of the nanograins dominate the behavior of the Fe grains, so the saturation magnetization is reduced by decreasing the particle size. During the precipitation process, a magnetic dead layer will form on the surface of Fe nanograins, severely reducing the magnetic moment and increasing the coercivity. ${ }^{9}$ As the aging time increases, more $\mathrm{Fe}$ nanograins with smaller magnetization will appear. Therefore, the saturation magnetization is decreasing with aging time.

Different concentrations of $\mathrm{Fe}$ yield different rates of magnetic variation. When the Fe concentration is small, the magnitude of its variation appears to be larger. The term $\Delta m_{s}\left[m_{s(\max )}-m_{s(\min )}\right]$ is divided by $m_{s(\max )}$. The values of $\Delta m_{s} / m_{s(\max )}=0.43,0.48$, and 0.71 for $x=0.5,0.4$, and 0.3 , respectively, were obtained. The solubility limit on the amount of $\mathrm{Bi}$ atoms that can be replaced by Fe atoms causes

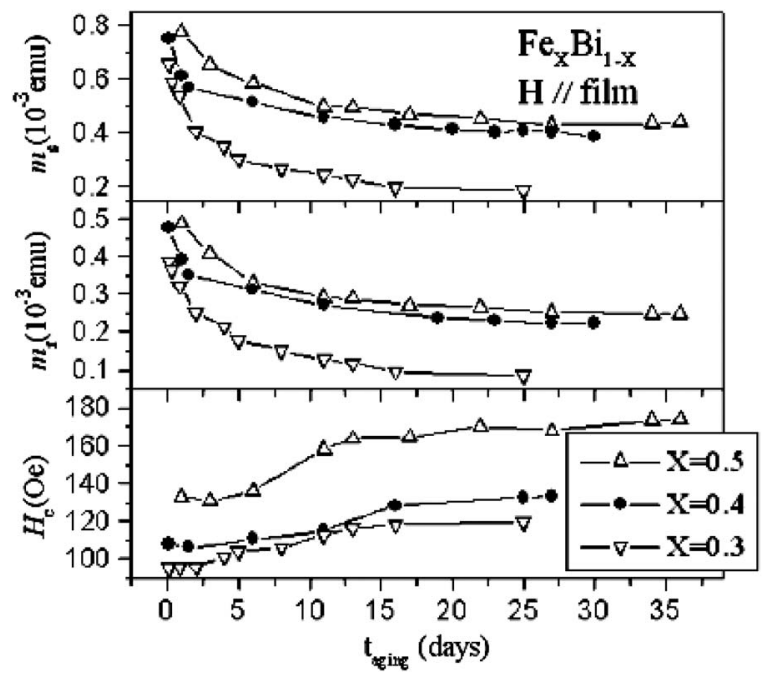

FIG. 4. Dependence of saturation magnetization $\left(m_{s}\right)$, remanence $\left(m_{r}\right)$, and coercivity field $\left(H_{c}\right)$ on aging time. the number of insoluble $\mathrm{Fe}$ atoms in $\mathrm{Bi}$ to increase with $\mathrm{Fe}$ concentration; these atoms do not participate in the precipitation reaction. Therefore, the fractional change in magnetism with aging seems small when $x$ is large.

A set of samples was sputtered with a protective layer of Ag of $0.5 \mu \mathrm{m}$ in thickness for comparison, to eliminate the effect of oxidation on the samples. Similar results were obtained; the values of $m_{s}$ and $m_{r}$ decrease and $H_{c}$ increases upon aging. Therefore oxidation is determined not to cause the time dependence of the magnetic properties. Actually the $\mathrm{Fe}-\mathrm{Bi}$ alloy is highly resistant to oxidation at room temperature. ${ }^{10}$ In addition, when the protective coated sample was annealed at $200{ }^{\circ} \mathrm{C}$ for $1 \mathrm{~h}$ in an $\mathrm{Ar}$ atmosphere, initially its $m_{s}$ and $m_{r}$ decreased rapidly. However, they gradually increased as the heat treatment was increased, perhaps because of the grain growth.

The same experiments were performed on $\mathrm{Co}-\mathrm{Bi}$; in these experiments, aging had a much weaker influence on the magnetic properties. The as-prepared sample did not exhibit any magnetic anisotropy. Co-Bi has a typical ferromagnetic hysteresis loop, without any linear magnetization due to anisotropy. Aging affects $\mathrm{Co}-\mathrm{Bi}$ more weakly than $\mathrm{Fe}-\mathrm{Bi}$. The variance of $m_{s}$ was estimated for films of different Co concentrations, whose $\Delta m_{s} / m_{s(\max )}$ were determined to be 0.043 , 0.078 , and 0.135 when $x=0.5,0.4$, and 0.3 . As for $\mathrm{Fe}-\mathrm{Bi}$, reducing the concentration of Co increased the variation, but the variation remained smaller than that for $\mathrm{Fe}-\mathrm{Bi}$.

In summary, metastable films of $\mathrm{Fe}-\mathrm{Bi}$ solid solution were prepared and unique time-dependent magnetic behaviors, which are unusual in most composite systems, were observed. The as-prepared $\mathrm{Fe}-\mathrm{Bi}$ was in the metastable solid solution phase and thus underwent natural aging. The $\mathrm{Fe}-\mathrm{Bi}$ solid solution exhibited strong out-of-plane magnetic anisotropic behavior, which disappeared after a long aging time. The development of the magnetic dead layer on the Fe particle surface caused by precipitation rapidly reduced saturation magnetization.

\section{ACKNOWLEDGMENTS}

The authors would like to thank the National Science Council of Taiwan, Contract No. NSC-93-2112-M-002-037 and the Ministry of Economic Affairs of the Republic of China, Taiwan, Contract No. MOEA 93-EC-17-A-08-S10006 for financially supporting this research.

${ }^{1}$ J. H. Hsu, J. T. Lee, C. R. Chang, and M. T. Lin, J. Magn. Magn. Mater. 226, 502 (2001).

${ }^{2}$ S. Honda and Y. Nagata, J. Appl. Phys. 93, 5538 (2003).

${ }^{3}$ N. Garcia, Y. H. Kao, and M. Strongin, Phys. Rev. B 5, 2029 (1972).

${ }^{4}$ R. E. Reed-Hill and R. Abbaschian, Physical Metallurgy Principles, 3rd ed. (PWS-Kent, Boston, MA, 1992).

${ }^{5}$ Q.-M. Chen, F.-Z. Cui, Y.-D. Fan, and H.-D. Li, J. Appl. Phys. 63, 2452 (1988).

${ }^{6}$ J. Q. Xiao, C. L. Chien, and A. Gavrin, J. Appl. Phys. 79, 5309 (1996).

${ }^{7}$ F. Z. Cui, Y. D. Fan, Y. Wang, A. M. Vredenberg, H. J. G. Draaisma, and R. Xu, J. Appl. Phys. 68, 701 (1990).

${ }^{8}$ Y. Yamada, T. Suzuki, and E. N. Abarra, IEEE Trans. Magn. 33, 3622 (1997); 34, 343 (1998).

${ }^{9}$ T. Taniyama, E. Ohta, T. Sato, and M. Takeda, Phys. Rev. B 55, 977 (1997).

${ }^{10}$ D. W. Forester, J. H. Schelleng, P. Lubitz, P. D'Antonio, and C. George, J. Appl. Phys. 53, 2240 (1982). 